\title{
Unconventional intronic splice site mutation in SCN5A associates with cardiac sodium channelopathy
}

\author{
T Rossenbacker, E Schollen, C Kuipéri, T J L de Ravel, K Devriendt, G Matthiijs, D Collen, \\ H Heidbüchel, P Carmeliet
}

J Med Genet 2005;42:e29 (http://www.jmedgenet.com/cgi/content/full/42/5/e29). doi: 10.1136/jmg.2004.029058

Background: Mutations in the cardiac sodium channel, SCN5A, have been associated with one type of long-QT syndrome, with isolated cardiac conduction defects and Brugada syndrome. The sodium channelopathies exhibit marked variation in clinical phenotypes. The mechanisms underlying the phenotypical diversity, however, remain unknown. Exonic SCN5A mutations can be detected in $20 \%$ of Brugada syndrome patients.

Results: An intronic mutation (c.4810+3_4810+6dupGGGT) in the SCN5A gene, located outside the consensus splice site, was detected in this study in a family with a highly variable clinical phenotype of Brugada syndrome and/or conduction disease and in a patient with Brugada syndrome. The mutation was not found in a control panel of 100 (200 alleles) ethnically matched normal control subjects. We provide in vivo and in vitro evidence that the mutation can disrupt the splice donor site, activate a cryptic splice site, and create a novel splice site. Notably, our data show that normal transcripts can be also derived from the mutant allele.

Conclusions: This is the first report of an unconventional intronic splice site mutation in the SCN5A gene leading to cardiac sodium channelopathy. We speculate that its phenotypical diversity might be determined by the ratio of normal/abnormal transcripts derived from the mutant allele.

M utations in the cardiac sodium channel, SCN5A (MIM 600163), have been linked with long-QT syndrome subtype $3^{1}$ (MIM 603830), with isolated cardiac conduction defect (ICCD) ${ }^{2}$ (MIM 113900), and with

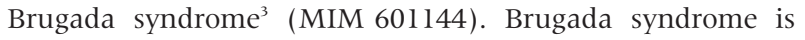
characterised by an ST-segment elevation in the right precordial leads (V1-V3), often accompanied by a right bundle branch block, and by a propensity for the development of ventricular arrhythmias. ${ }^{4}$ The phenotypic expression of the sodium channelopathies is complicated at three levels. First, not all carriers of a pathogenic mutation manifest a phenotype. ${ }^{5}$ Second, not all SCN5A mutation carriers manifest an identical phenotype, that is, the clinical phenotype of Brugada syndrome is highly variable, ranging from asymptomatic carriers to sudden cardiac death. ${ }^{6}$ Single SCN5A mutations can also lead to overlapping phenotypes. For instance, the G1460R mutation in SCN5A is associated with Brugada syndrome and ICCD in the same family. ${ }^{7}$ Third, distinct clinical disorders share the same clinical features, that is, conduction abnormalities are part of the normal clinical spectrum of Brugada syndrome ${ }^{8}$ and are also obligatory for the diagnosis of ICCD. It has been proposed that as yet unidentified modifiers may modulate both the expression and the severity of a given clinical phenotype. ${ }^{9}$ In this study, we sought to determine the role of a novel SCN5A mutation in the variable phenotypic expression of the disease.

Mutations in coding regions of the SCN5A gene can only be found in approximately $20 \%$ of Brugada syndrome patients. ${ }^{6}$ It is assumed that defects in as yet unidentified genes are responsible for the remainder of Brugada syndrome cases. In this study, an unconventional intronic splice error in the SCN5A gene was investigated as the cause of a sodium channelopathy with a diverse phenotype.

\section{METHODS}

\section{Clinical investigation}

The study is based on one western European family (proband III:1) and one western European patient (patient A). Proband III: 1 and patient A underwent full clinical and electrophysiological investigation, including programmed electrical stimulation (PES) with up to three ventricular extra-stimuli (ES) at two drive cycle lengths. Family members of patient III: 1 were evaluated with ECG and/or with SAECG and/or with procaïnamide challenge ( $10 \mathrm{mg} / \mathrm{kg} \mathrm{IV})$. A PR duration of $\geqslant 200 \mathrm{~ms}$ or a QRS duration of $\geqslant 115 \mathrm{~ms}$ was considered prolonged. An $\mathrm{AH}$ interval $\geqslant 120 \mathrm{~ms}$ (in sinus rate) or a Wenckebach cycle length $\geqslant 400 \mathrm{~ms}$ (that is, 150/min) (baseline state) was considered indicative for atrioventricular nodal conduction slowing. An HV interval $\geqslant 60 \mathrm{~ms}$ was considered as proof for His-Purkinje conduction slowing.

\section{Mutation analysis}

Genomic DNA was prepared from peripheral blood lymphocytes by standard methods. The entire coding region of the SCN5A gene was amplified by PCR and analysed by denaturing high performance liquid chromatography (DHPLC; Transgenomics, Crewe, UK) and subsequent sequencing using fluorescent dye chemistry (BigDye Terminator v3.1 Cycle Sequencing Kit, Applied Biosystems, Lennik, Belgium) and an ABI-3100 genetic analyser (Applied Biosystems). Absence or presence of the mutation and single nucleotide polymorphisms (SNPs) in family members was assessed by DHPLC.

\section{cDNA analysis}

Total RNA was isolated with Trizol (Invitrogen, Merelbeke, Belgium) from lymphocytes according to the manufacturer's protocol. cDNA was prepared using $5 \mu \mathrm{g}$ total RNA in a total volume of $19.5 \mu \mathrm{l}$ with SuperscriptII RNAse H-reverse transcriptase and oligo-dT priming (Invitrogen). The SCN5A transcript was amplified in two successive PCR reactions with nested PCR primers: the first amplicon was $1800 \mathrm{bp}$ long

Abbreviations: AV, atrioventricular; DHPLC, denaturing high performance liquid chromatography; EPS, electrophysiological study; ES, extra-stimuli; ICCD, isolated cardiac conduction defect; PES, programmed electrical stimulation; SNPs, single nucleotide polymorphisms; WT, wildtype 


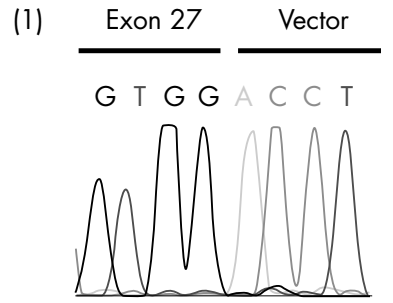

(3)

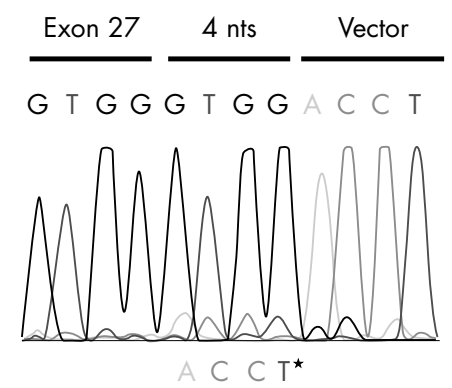

(2)
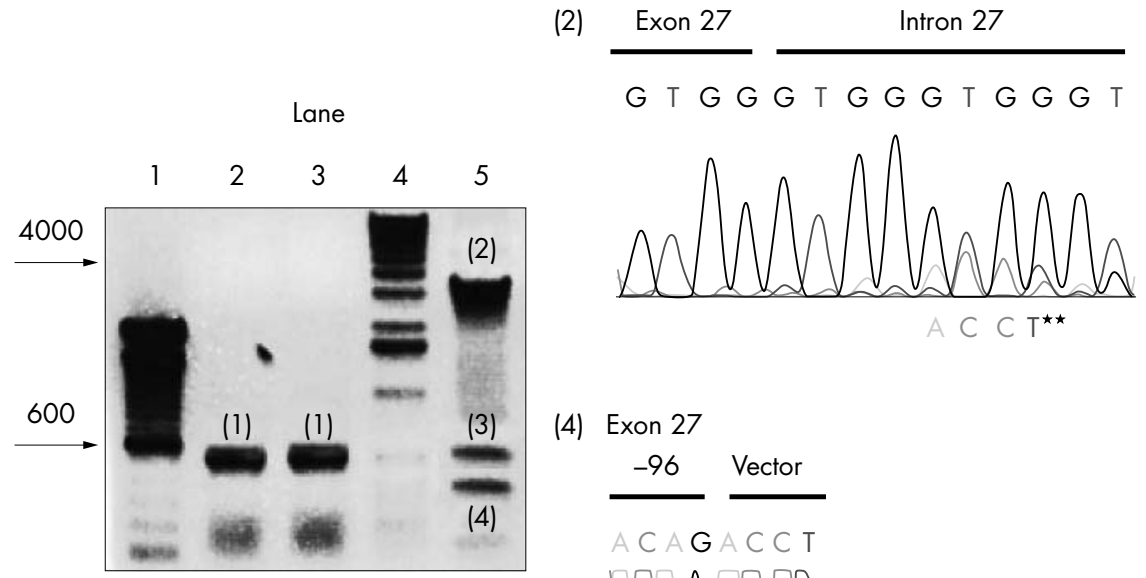

(4) Exon 27

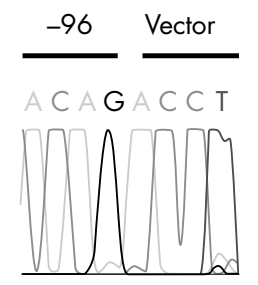

Figure 1 Reverse transcriptase-PCR with vector primers SD6 and SA2 (see fig 3) on COS-7 cells transfected with WT and mutant pSPL3. Amplification products were characterised by direct sequencing. Lane 1: 100 bp DNA ladder (Eurogentec, Seraing, Belgium). Lanes 2 and 3: WT. Lane 4: 1 kb DNA adder (Eurogentec). Lane 5: mutant. One WT band of $532 \mathrm{bp}$ can be seen in lanes 2 and 3. Direct sequencing indicated correct splicing (see also fig 3). For the mutant, three bands of 2930,536, and 436 bp can be seen. Sequencing of the upper band revealed transcript 1 (see also fig 3). In the background, the sequence of transcript 3 can be detected (indicated by ${ }^{* *}$ ). Note that PCR product was PCR purified and not prepared by gel extraction. Sequencing of the middle band detected transcript 3 (see also fig 3). Notably, the sequence of a WT transcript can be noticed in background (indicated by *). Sequencing of the lower band revealed transcript 2 (see also fig 3).

(from c.3819 to c.5618) with primers F: 5'-GCT CGA CTT CCT CAT CGT AGA-3' and R: 5'-ATC TTC AGG GCG TCC AT-3'; the nested fragment was 945 bp long (from c.4636 to c.5580) with primers F: 5'-TGG TGG AGA CAG ATG ACC AA-3' and R: 5'-CCT TTT GGT GAA GGC AAA GA-3'. PCR was performed in $50 \mu \mathrm{l}$ with $1 \mu \mathrm{l}$ cDNA in the first reaction and $2 \mu \mathrm{l}$ of the first PCR product in the second reaction using regular Taq polymerase and PCR buffer (Roche Diagnostics, Indianapolis, IN). The reaction conditions were: $95^{\circ} \mathrm{C}, 2 \mathrm{~min}$; $\left(95^{\circ} \mathrm{C}, 20 \mathrm{~s} ; 65^{\circ} \mathrm{C}, 20 \mathrm{~s}\left(-1{ }^{\circ} \mathrm{C} / \mathrm{Cycle}\right) ; 72^{\circ} \mathrm{C}, 1 \mathrm{~min}\right) \times 10 ;\left(95^{\circ} \mathrm{C}\right.$, $\left.20 \mathrm{~s} ; 55^{\circ} \mathrm{C}, 20 \mathrm{~s} ; 72^{\circ} \mathrm{C}, 1 \mathrm{~min}\right) \times 27 ; 72^{\circ} \mathrm{C}, 5 \mathrm{~min} ; 4^{\circ} \mathrm{C}$, hold. The amplicons were analysed by direct sequencing using BigDye Terminator v3.1 Cycle Sequencing Kit (Applied Biosystems) and analysis on ABI-3100 (Applied Biosystems).

\section{Exon trapping experiments}

Using DNA from proband III:1 as template, harbouring one wildtype (WT) and one mutant allele, a 933 (WT) and 937 (mutant) base pair (bp) SCN5A genomic fragment, containing exon 27 with $347 \mathrm{bp}$ of $5^{\prime}$ and $315 \mathrm{bp}$ of $3^{\prime}$ intronic flanking sequences, was generated (forward primer F: 5-TTG AGC CTT CAG ACT GTG GGA-3' and reverse primer R: 5' -TTC CCA GAC TCA TCC TTG AAG-3'), cloned into pGEM-T easy vector (Promega, Leiden, The Netherlands), and subsequently subcloned into pSPL3 exon trapping vector (kindly donated by Dr JJ Schott, Nantes, France). Direct sequencing analysis of recombinant clones identified WT and mutant pSPL3 plasmids, without evidence of additional PCR induced mutations. WT or mutant plasmids were transiently transfected into African green monkey cells (COS-7) using Superfect Reagens (Westburg, Leusden, The Netherlands). COS-7 cells were cultured in Dulbecco's modified Eagle's medium supplemented with $10 \%$ fetal bovine serum, $1 \%$ penicillin-streptomycin, and $1 \%$ glutamine in a humidified, $5 \% \mathrm{CO}_{2}$ incubator at $37^{\circ} \mathrm{C}$. At $48 \mathrm{~h}$ post-transfection, total RNA was extracted with Trizol and retrotranscribed with SD6 oligonucleotide according to the recommendations of the manufacturer (Exon Trapping System, Invitrogen,
Merelbeke, Belgium). Amplification products, obtained by PCR with vector primers SD6 and SA2 (donated by Dr JJ Schott), were separated on a $1 \%$ TAE agarose gel. Amplification products corresponding to a size of $\sim 536 \mathrm{bp}$ band (see fig 1) were subcloned into pGEM-T easy vector (Promega) and characterised by direct sequencing.

\section{RESULTS}

\section{Association of splice site mutation with cardiac sodium channelopathy}

Female proband III: 1 presented at the age of 15 with syncope evoked by atrial flutter with 1:1 conduction. Baseline ECG recording showed Brugada syndrome phenotype, first degree atrioventricular (AV) block, and intraventricular conduction disturbances (fig 2C). An electrophysiological study (EPS) noted diffuse conduction disturbances at AV nodal and ventricular level. The patient underwent successful catheter ablation of the flutter without later recurrence. PES induced polymorphic ventricular tachycardia with one and two ES and ventricular fibrillation with three ES. An implantable cardioverter defibrillator was inserted. After 2 years of follow up no appropriate shocks were delivered. The family pedigree is shown in fig $2 \mathrm{~A}$ and the clinical data of family members are given in table 1 . As can be appreciated, clinical phenotypes are highly variable, ranging from a benign presentation with a subtle conduction defect to a more aggressive variant of the disease. Patients with no Brugada syndrome features but with aberrant PR and/or QRS durations (for example, patient II:2; see ECG in fig 2D), were considered to be ICCD patients. Patients with either ECG Brugada syndrome features or positive class I challenge with or without abnormal conduction parameters were diagnosed as patients affected with Brugada syndrome.

Patient A was asymptomatic. A typical Brugada ECG pattern was seen in the setting of a pre-operative screening at age 62 (fig 2E). PES (up to three ES) failed to elicit arrhythmias and no conduction abnormalities were seen 
A

C

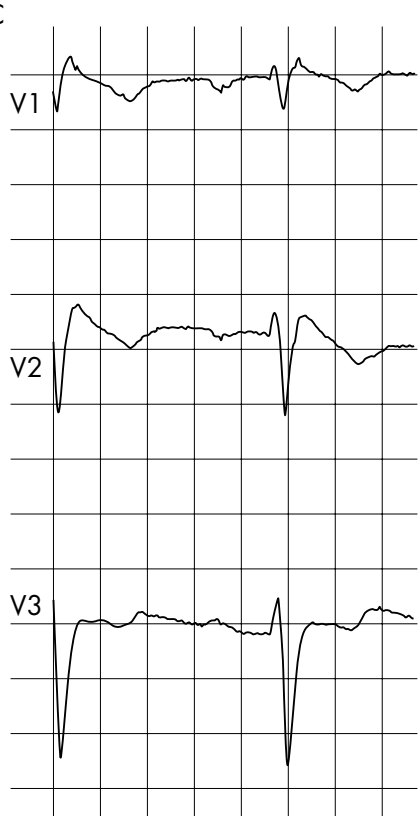

Proband III: 1
D

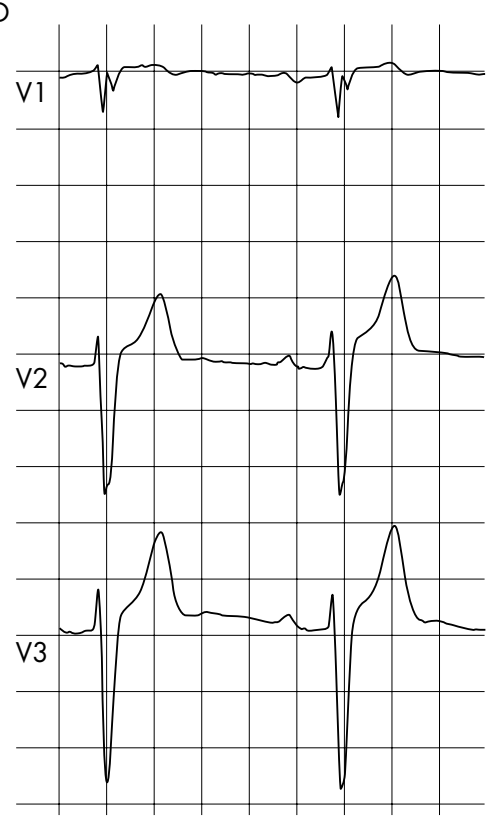

Patient II:2
B

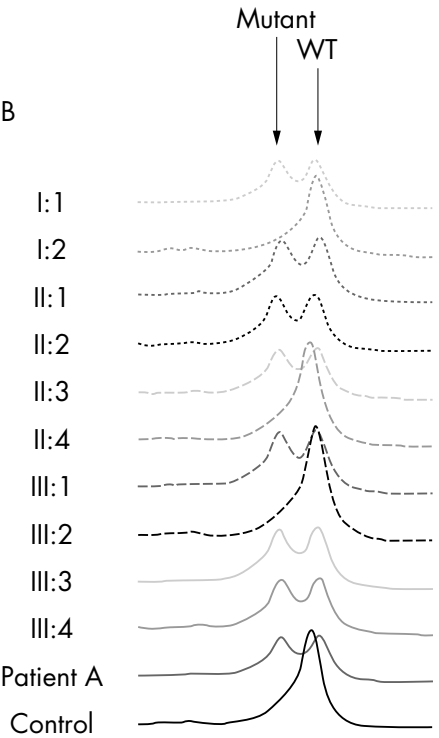

Figure 2 (A) Family pedigree of proband III: 1. Squares: males; circles: females; filled symbols: mutation carriers. See table 1 for clinical information. (B) Co-segregation of the mutation, assessed by DHPLC. (C-E) ECG: precordial leads V1-V3 of proband III: 1, patient II:2, and patient A, respectively In (C) and (E) but not in (D), a Brugada ECG can be seen. PR is prolonged in (C) and (D): 285 and $210 \mathrm{~ms}$, respectively. QRS is prolonged in (C) and (E): 140 and $115 \mathrm{~ms}$, respectively.

during EPS. Microsatellite marker data and haplotype analysis based on detection of SNPs in the SCN5A gene were indicative of a common ancestor for proband III: 1 and patient A. The following SNPs were analysed: IVS2-24C/T, IVS16-6C/ T, c. $3183 \mathrm{G} / \mathrm{A}$, and IVS19+72T/C. The haplotype shared between proband III: 1 and patient A was IVS2-24T (2\%)/ IVS16-6T (4\%)/c.3183A (14\%)/IVS19+72C. The frequencies of the specified allele in a control population of 100 healthy subjects is given between brackets. Considering the low frequency of these SNPs, the fact that each of these individuals carries the same haplotype strongly suggests a common ancestor origin. Additionally, both patients shared the same length for microsatellite markers D3S1298 (197 bp) and D3S3593 (214 bp).
Mutation detection analysis of the SCN5A gene in the proband and in patient A only revealed a duplication of 4 bases in the splice donor of intron 27 (c.4810+3_4810+6dupGGGT) besides several additional intronic SNPs, more distantly removed from the splice site. The aberration co-segregated in affected family members (fig 2B) and was not found in a control panel of 100 (200 alleles) ethnically matched normal control subjects.

\section{Abnormal splicing by the c.4810+3_4810+6dupGGGT} allele

According to several splice prediction programs (data not shown), the splice site of intron 27 is a weak splice donor. The duplication of GGGT in a repeat of two copies of GGGT, 
Table 1 Clinical data

\begin{tabular}{|c|c|c|c|c|c|c|c|c|c|c|c|c|c|c|}
\hline \multirow[b]{2}{*}{ Patient } & \multirow[b]{2}{*}{ Age (years)* } & \multirow[b]{2}{*}{ Sex } & \multirow[b]{2}{*}{$\Sigma$} & \multicolumn{5}{|c|}{ Baseline ECG } & \multirow[b]{2}{*}{ LP } & \multirow[b]{2}{*}{ Class I } & \multicolumn{4}{|l|}{ EPS } \\
\hline & & & & $\mathrm{Br}$ & PR & QRS & Axis & QTc & & & $\mathrm{AH}$ & HV & WCL & I \\
\hline $\mathrm{l}: 2$ & 66 & $\mathrm{~F}$ & No & $N$ & 256 & 100 & $\mathrm{Nl}$ & & & & & & & \\
\hline II:1 & 37 & $M$ & Afib & $\mathrm{N}$ & 220 & 125 & $\mathrm{Nl}$ & 431 & $P$ & $\mathrm{~N}$ & & & & \\
\hline$\|: 2$ & 42 & $M$ & No & $\mathrm{N}$ & 210 & 116 & Right & 390 & & & & & & \\
\hline II:3 & 34 & $M$ & No & $\mathrm{N}$ & 258 & 110 & $\mathrm{Nl}$ & & & & & & & \\
\hline III: 1 & 20 & $\mathrm{~F}$ & Aflut & $P$ & 285 & 140 & Right & 449 & & & 140 & 60 & $640 \dagger$ & $P$ \\
\hline III:2 & 13 & M & No & $\mathrm{N}$ & 165 & 95 & $\mathrm{Nl}$ & 405 & & $\mathrm{~N}$ & & & & \\
\hline III:3 & 13 & $M$ & No & $N$ & 205 & 120 & Right & 420 & $P$ & $N$ & & & & \\
\hline III:4 & 11 & $\mathrm{~F}$ & No & $N$ & 170 & 105 & $\mathrm{Nl}$ & 403 & $P$ & $P$ & & & & \\
\hline A & 62 & $\mathrm{~F}$ & No & $P$ & 230 & 115 & †Left & 410 & $P$ & & 120 & 60 & $480 \S$ & N \\
\hline
\end{tabular}

Afib, atrial fibrillation; Aflut, atrial flutter; Br, Brugada type 1 ECG; EPS, electrophysiological study; $F$, female; I, inducibility; LP, late potentials; M, male; N, negative; NI: normal; $\mathrm{P}$, positive; $\Sigma$, symptoms; WCL, Wenckebach cycle length.

${ }^{*}$ At time of investigation; $\dagger, \S 1: 1$ antegrade conduction via fast pathway till cycle length of $>1000$ and 520 ms, respectively; $\ddagger$ left anterior hemiblock.

spanning the splice site, leaves the first six bases of the donor splice site unaffected. Because of the special nature of the duplication, we decided to test the pathogenic effect of this aberration on the correct splicing of exon 27.

Although the SCN5A gene is supposed to be uniquely expressed in heart, we were able to amplify SCN5A transcripts in lymphocytes. While in control cells only the WT transcript was amplified, a WT and an aberrant transcript (corresponding to transcript 2, fig 3) were detected in lymphocytes from proband III: 1 and patient A. Unfortunately, the level of these transcripts in lymphocytes was too low to permit reliable relative quantification of each transcript.

To exclude aberrant splicing due to illegitimate transcription, we tested the effect of the mutation in an in vitro exon trapping experiment. Vectors harbouring the WT or the mutant genomic fragment, containing exon 27 and at least $300 \mathrm{bp}$ flanking intronic sequences, were transfected in COS-7 cells. As indicated in figs 1 and 3, splicing in COS-7 cells, transfected with WT vector, consistently generated WT transcript, whereas splicing in cells transfected with the mutant vector produced three alternatively spliced forms (transcripts 1, 2, and 3; fig 3). In transcript 1, the $5^{\prime}$ splice donor site is not recognised and no splicing occurs at this site, resulting in incorporation of intronic sequences in this transcript. An additional number of 95 amino acids will be translated from the intronic sequence before a stop codon terminates translation. These additional residues are completely unrelated to the native sequence of the sodium channel. Transcript 2 is spliced from a cryptic splice donor site within exon 27, resulting in a deletion of the $3^{\prime}$-most 96 bases of exon 27. This causes an in-frame deletion of amino acids 1572-1604. Considering the extent of the deletion which covers fragments of segments 2 and 3 of domain IV, it is likely that this will have an impact on protein folding and function. In transcript 3, a novel splice donor site is used at the intronic GT, 3' of exon 27, resulting in the incorporation of four additional nucleotides. This causes a frame-shift Gl604fsi83 (that is, glycine as last unaffected amino acid (AA) at position 1604 followed by 183 AAs before termination) which leads to a non-functional SCN5A channel lacking part of domain IV and the C terminus. Thus, the nature of each of these transcript anomalies is expected to encode either no or only non-functional sodium channels.

\section{Normal splicing by the c.4810+3_4810+6dupGGGT allele}

As the duplication leaves the original consensus donor splice site unaffected, we also assessed whether correct "WT-like" splicing of the mutant allele still occurred. Since the WT transcript is only four nucleotides shorter than mutant transcript 3, it cannot be easily separated by conventional agarose gel electrophoresis. A low signal of mutant transcript 3 in the sequence analysis (fig 1) suggested, however, the presence of WT transcript in cells transfected with the mutant construct. We therefore subcloned the PCR amplification products with an approximate size of 530-540 bp (fig 1) and sequenced 59 clones. Out of those 59 clones, two clones contained the WT transcript (data not shown), indicating that the mutant allele is indeed capable of encoding a WT channel, though at a reduced level.

\section{DISCUSSION}

In this report, an unconventional splice site mutation in SCN5A is described as the cause of a sodium channelopathy. Ten base pairs $(-4$ to +6$)$ around the 5' exon-intron boundary are essential for normal splicing and constitute the donor splice site. ${ }^{10}$ Sequence alterations at these locations are potentially disease causing. Previous splice site mutations, associated with ICCD or Brugada syndrome, have been predicted to cause exon skipping by disruption of the splice site. ${ }^{2} 3^{11-14}$ Exon skipping causes either a frameshift and a premature stop codon or a deletion of the region encoded by the exon. As evidenced by an in vitro approach, the mutation described here can simultaneously disrupt the splice donor site (mutant transcript 1), can activate a cryptic splice site (mutant transcript 2), and can create a novel donor site (mutant transcript 3). To study the consequences of the splice mutation on the generation of SCN5A transcripts in a human cell line, we used lymphocytes as heart tissue of affected patients was unavailable. Mutant transcript 2 could be isolated in proband III:1 and in patient A but not in control patients. We are well aware of the problem of illegitimate transcription, which could lead to aberrant splice products of any gene in any cell type. However: (i) the same splice error was seen in both patients; (ii) the splice error was not detected in control patients; (iii) data were reproducible in subsequent experiments; and (iv) the splicing abnormality, detected in the patient's lymphocytes, was also found in the exon trapping experiments. The fact that mutant transcript 1 and 3 were found in transfected COS-7 cells but not in lymphocytes may be explained by (i) differences in the spliceosome between human lymphocytes and COS-7 cells and (ii) by the absence of the native acceptor splice site of intron 27 in the experiments with COS-7 cells.

The duplication leaves the consensus donor splice site unaffected and, thus, correct "WT-like" splicing of the mutant allele could, theoretically, still occur. This question can, however, not be easily addressed by analysing lymphocytes from affected heterozygous individuals, as they already carry a single WT allele. However, exon trapping experiments with the isolated mutant allele revealed that a WT transcript was indeed generated from the mutant allele. It can thus be speculated that the ratio of normal/abnormal transcripts and, 
A Wild type

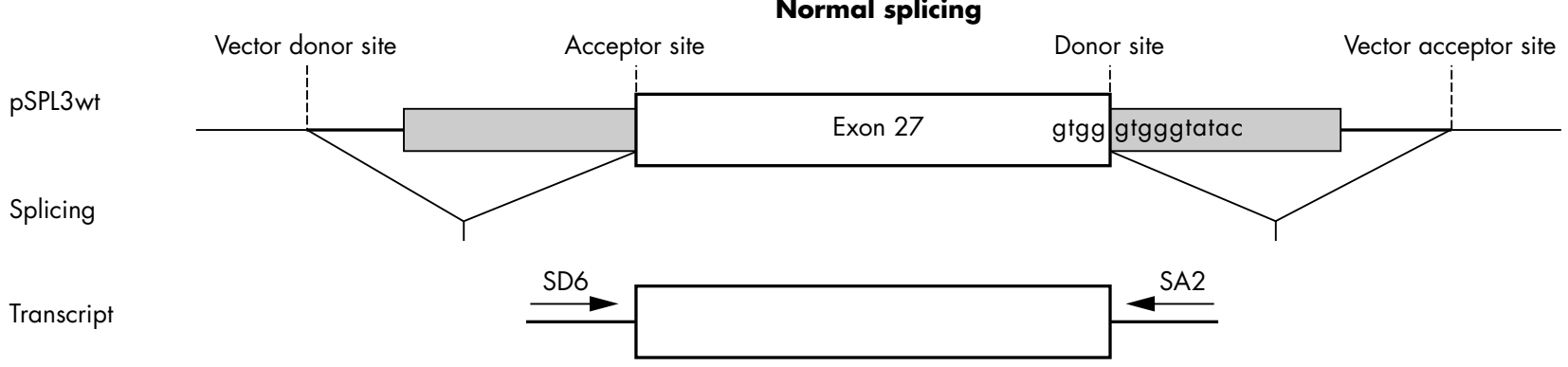

B Mutant
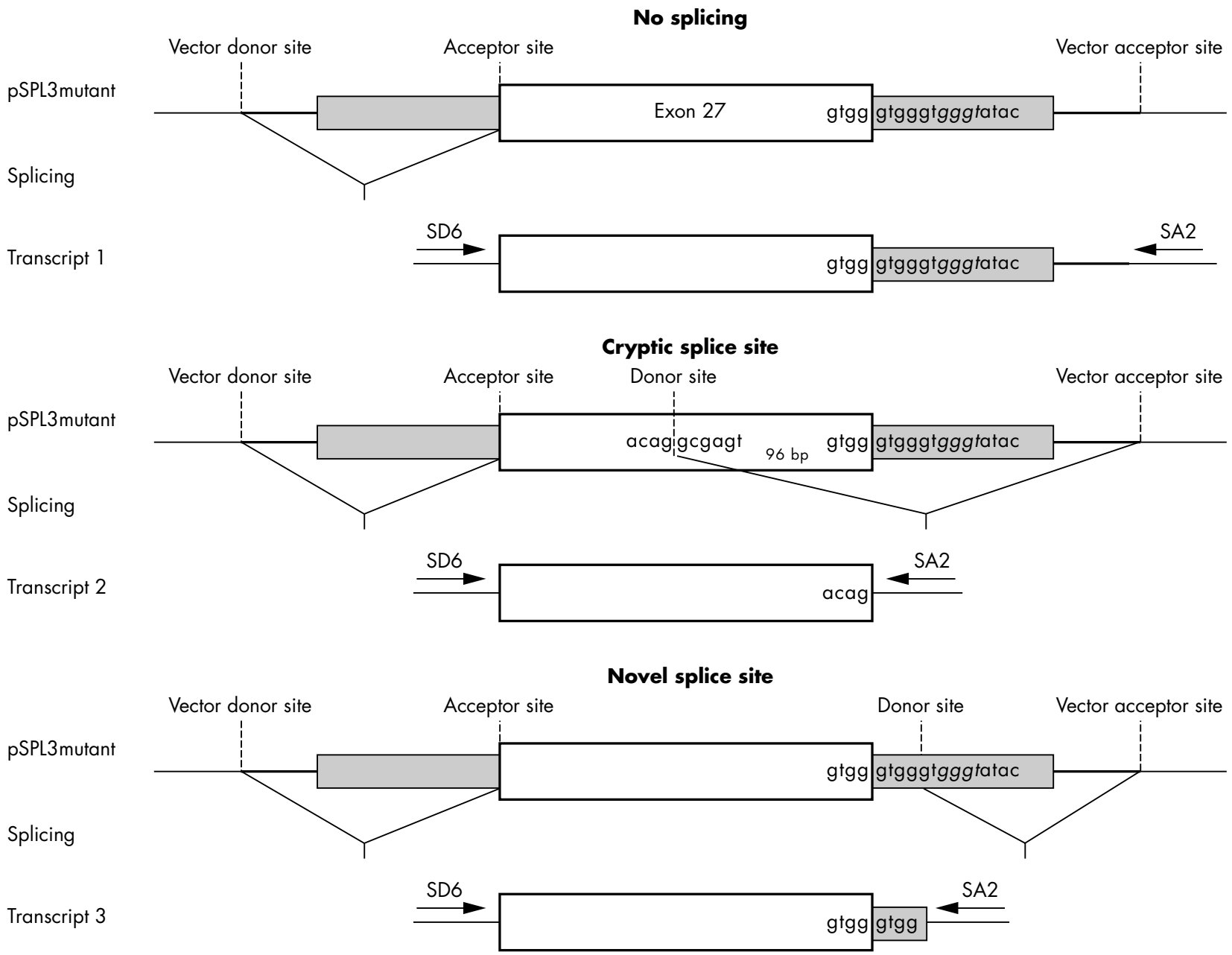

Figure 3 (A) WT plasmid generates WT transcripts. (B) Mutant plasmid generates three aberrant transcripts (1-3). WT (A) and mutant (B) exon 27 and part of flanking introns (347 and $315 \mathrm{bp}$ ) were subcloned in exon trapping vector pSPL3 and transfected in COS-7 cells. Transcripts are derived from the pairing of vector and cloned splice signals. In transcript 1, the 5' splice site is not recognised and no splicing occurs at this site. In transcript 2, a cryptic splice site in exon 27 becomes activated which causes a deletion of 96 bases in exon 27. In transcript 3, a novel splice site is used, that is, second intronic GT which leads to incorporation of four extra bases in the transcript. Mutation is indicated in italic. SD6 and SA2 are vector primers used for PCR (see fig 1).

consequently, the ratio of functional/non-functional channels, might influence the phenotypic expression of the disease in affected family members. At least theoretically, it is conceivable that the presence of some WT channels in affected individuals might result in a milder phenotype, whereas the complete absence of WT channels might be expected to yield the most severe clinical phenotype.
In conclusion, the c. $4810+34810+6$ dupGGGT SCN5A is a novel intronic mutation that leads to aberrant splicing. Lymphocytes could be used to assess the functional effect of the mutation and constitute an interesting approach for the study of other SCN5A splice site mutations. The mutation is located outside the consensus splice site. Our findings point to the role of intronic nucleotides other than at 
invariant donor sites. Whether a given ratio of normal/ abnormal transcripts might contribute to the variation in phenotypic characteristics in different family members, awaits further confirmation by investigation of heart tissue of affected patients.

\section{Authors' affiliations}

T Rossenbacker, D Collen, P Carmeliet, Centre for Transgene

Technology and Gene Therapy, Flanders Interuniversitary Institute for

Biotechnology, KULeuven, Leuven, Belgium

E Schollen, C Kuipéri, T J L de Ravel, K Devriendt, G Matthijs, Centre for

Human Genetics, UZLeuven, Leuven, Belgium

H Heidbüchel, Centre for Hereditary Heart Diseases, KULeuven, Leuven, Belgium

TR is a research assistant of the Fund for Scientific Research Flanders (FWO). This work was supported in part by a grant from FWO (\#G.0281.03)

Competing interests: none declared

Ethics approval: The study was performed in accordance with recommendations of the Ethics Committee of the University Hospital Leuven.

Written informed consent to participate in the study was obtained from all subjects or their legal representatives.

Correspondence to: Dr P Carmeliet, Centre for Transgene Technology and Gene Therapy, Flanders Interuniversitary Institute for Biotechnology, KULeuven, Campus Gasthuisberg, Herestraat 49, B-3000, Leuven, Belgium; peter.carmeliet@med.kuleuven.ac.be

Revised version received 4 January 2005

Accepted for publication 6 January 2005

\section{REFERENCES}

1 Wang Q, Shen J, Splawski I, Atkinson D, Li Z, Robinson JL, Moss AJ, Towbin JA, Keating MT. SCN5A mutations associated with an inherited cardiac arrhythmia, long QT syndrome. Cell 1995;80:805-11.
2 Schott JJ, Alshinawi C, Kyndt F, Probst V Hoorntie TM, Hulsbeek M, Wilde AA, Escande D, Mannens MM, Le Marec H. Cardiac conduction defects associate with mutations in SCN5A. Nat Genet 1999;23:20-1.

3 Chen Q, Kirsch GE, Zhang D, Brugada R, Brugada J, Brugada $P$, Potenza D, Moya A, Borggrefe M, Breithardt G, Ortiz-Lopez R, Wang Z, Antzelevitch C, O'Brien RE, Schulze-Bahr E, Keating MT, Towbin JA, Wang Q. Genetic basis and molecular mechanism for idiopathic ventricular fibrillation. Nature 1998:392:293-6.

4 Brugada P, Brugada J. Right bundle branch block, persistent ST segment elevation and sudden cardiac death: a distinct clinical and electrocardiographic syndrome. A multicenter report. J Am Coll Cardiol 1992;20:1391-6.

5 Priori SG, Napolitano C, Schwartz PJ. Low penetrance in the long-QT syndrome: clinical impact. Circulation 1999;99:529-33.

6 Antzelevitch C, Brugada P, Brugada J, Brugada R, Towbin JA, Nademanee K. Brugada syndrome: 1992-2002: a historical perspective. J Am Coll Cardiol 2003:41:1665-71.

7 Kyndt F, Probst V, Potet F, Demolombe S, Chevallier JC, Baro I, Moisan JP, Boisseau P, Schott JJ, Escande D, Le Marec H. Novel SCN5A mutation leading either to isolated cardiac conduction defect or Brugada syndrome in a large French family. Circulation 2001;104:3081-6.

8 Wilde AA, Antzelevitch C, Borggrefe M, Brugada J, Brugada R, Brugada P, Corrado D, Haver RN, Kass RS, Nademanee K, Priori SG, Towbin JA. Proposed diagnostic criteria for the Brugada syndrome: consensus report. Circulation 2002;106:2514-9.

9 Priori SG. Inherited arrhythmogenic diseases: the complexity beyond monogenic disorders. Circ Res 2004;94:140-5.

10 Padgett RA, Grabowski PJ, Konarska MM, Seiler S, Sharp PA. Splicing of messenger RNA precursors. Annu Rev Biochem 1986:55:1119-50.

11 Rook MB, Bezzina Alshinawi C, Groenewegen WA, van Gelder IC, van Ginneken AC, Jongsma HJ, Mannens MM, Wilde AA. Human SCN5A gene mutations alter cardiac sodium channel kinetics and are associated with the Brugada syndrome. Cardiovasc Res 1999;44:507-17.

12 Vatta M, Dumaine R, Antzelevitch C, Brugada R, Li H, Bowles NE, Nademanee K, Brugada J, Brugada P, Towbin JA. Novel mutations in domain I of SCN5A cause Brugada syndrome. Mol Genet Metab 2002;75:317-24.

13 Smits JP, Eckardt L, Probst V, Bezzina CR, Schott JJ, Remme CA Haverkamp W, Breithardt G, Escande D, Schulze-Bahr E, LeMarec H, Wilde AA. Genotype-phenotype relationship in Brugada syndrome: electrocardiographic features differentiate SCN5A-related patients from nonSCN5A-related patients. J Am Coll Cardiol 2002;40:350-6.

14 Priori SG, Napolitano C, Gasparini M, Pappone C, Della Bella P, Giordano U, Bloise R, Giustetto C, De Nardis R, Grillo M, Ronchetti E, Faggiano G, Nastoli J. Natural history of Brugada syndrome: insights for risk stratification and management. Circulation 2002;105:1342-7. 